\title{
VLADIMIR NAUMOVICH GRIBOV: PIECES OF BIOGRAPHY*
}

\author{
Ya. I. AZIMOV \\ Petersburg Nuclear Physics Institute, \\ National Research Center "Kurchatov Institute", \\ Gatchina, 188300, Russia \\ E-mail: azimov@thd.pnpi.spb.ru
}

\begin{abstract}
The talk presents the main lines of biography of the prominent physicist and bright personality. Also given is a necessarily brief description of Gribov's scientific work.
\end{abstract}

This talk I would like to begin with some personal recollections. I was lucky to work with V.N. Gribov for 20 years. It was not under Gribov, but just with. We have several common papers, several of my papers were made according to Gribov's suggestions. But most of my work were formally independent of Gribov. Nevertheless, all my work of those 20 years, in more or less detail, went through discussions with Gribov.

Of course, physics was not the only interest of Gribov, but, undoubtedly, it was his main interest producing very strong emotions. One could discuss with Gribov various physics problems, even rather far from his own studies (a couple of most bright examples will be mentioned below). But those discussions were not easy. Gribov was hearing very attentively and was ready to immediately jump into battle if something seemed incorrect to him. He had a rare feature: his arguments were very hard to reject even when his position appeared unjust after all (I think nobody can be just in all cases). The same situation was both in private discussions and at seminars. That is why talks at Gribov's seminar were very difficult for speakers. As a result, some people feared to speak at his seminar. However, if a person were sufficiently brave, the talk appeared very useful for the speaker. After

\footnotetext{
*Based on the invited talk at the 4th V.N. Gribov Memorial Workshop "Theoretical Physics of XXI Century" (Gribov-85), 17-20 June, 2015, Chernogolovka, Russia. To appear in the Proceedings.
} 
such trial the author himself began to better understand his own work and results.

Gribov's name and his papers (at least some of them) are well known today. But his biography is less known. Here I present its main lines.

Vladimir (Russian nickname Volodya) Gribov was born on March 25, 1930 in Leningrad (St.Petersburg, before 1914 and now). His farther died in 1938, at the time of the Great Terror in the Soviet Union. "Luckily", his death was the result of a decease, and not because of repression (in which case the whole family could be repressed as well). But the situation was not simple: the mother stayed alone with two small children, Volodya and his younger sister.

Gribov's mother worked in one of Leningrad theaters (not as an actress). When Germany attacked the Soviet Union in 1941, the family was evacuated from Leningrad together with the theater. They were moving with the theater over Siberia, Far East, Urals. Nevertheless, Volodya was continuing his school education, started in 1937, without any delay. Only in summer 1945 the family was able to return to Leningrad (to enter the city after the blockade, every person needed to have a special permit). In 1947 Volodya finished here the school course. The natural question arose: what to do after that?

Volodya was growing up in theatrical environment, and he dreamed to become an actor, best of all, a cinema actor. However, when being in senior school classes, he had a possibility to expose himself to filming. As appeared, under camera he became "frozen" and lost his natural mobility. After that, one of professional actors advised him to choose some other speciality. At school, Volodya was quite successful in physics and mathematics. He preferred the former.

In 1947 Gribov enrolled in Physical Faculty of the Leningrad University. He was a student together with D.V. Volkov (later, the full member of the Ukrainian Academy of Sciences) and G.M. Eliashberg (now the full member of the Russian Academy of Sciences, the chief researcher of the Landau Institute for Theoretical Physics). In 1950 Volkov was included into the special group to study nuclear physics in more detail, and in 1951 was transferred to the Kharkov University. Gribov also wished to be in that group, but was rejected.

In 1952 Gribov presented his diploma thesis considering interaction of two electrons in Quantum Electrodynamics (under supervision of Yu.V. Novozhilov). It was evaluated very high. According to decision of the examination commission, Bulletin of the Leningrad University pub- 
lished the paper ${ }^{1}$ based on this diploma work. It was the first publication signed by V.N. Gribov. Thus, in summer 1952 he was graduated from the University with diploma cum laude.

The year 1952 in the Soviet Union was not favorable for persons of jewish origin, one of which was Vladimir Naumovich Gribov. It was the last year for the trial of the Jewish Antifashist Committee (after its end, more than 100 persons were sentenced, more than 20 of them were shot). It was also the preparatory year for the "case of physicians" ("killers in white coats") which was officially opened in January 1953. In such situation the only job available for Gribov, a young physicist with diploma cum laude, appeared the position of a physics teacher in an evening school, organized for working people who could not, by any reason, complete their school education in the childhood. The salary was low, and year later Gribov found additional part-time work.

Nevertheless, Volodya wished and was continuing to do science. He was able to contact with Professor L.E. Gurevich. To the beginning of 1954, they together prepared two papers, on properties of matter in external fields, electric and gravitation. The papers were submitted to Journal of Experimental and Theoretical Physics and accepted for publication ${ }^{2,3}$. In addition, Gribov participated in the theoretical seminar of the Leninigrad Physico-Technical Institute (now the A.F. Ioffe Physico-Technical Institute, further PTI) headed by I.M. Shmushkevich and K.A. Ter-Martirosyan.

At last, in May 1954 (after Stalin's death and extinction of the "case of physicians"), Gribov was able to be employed by PTI as the senior laboratory assistant in the group for nuclear theory. It was headed by I.M. Shmushkevich, who was simultaneously an informal head of the Theoretical Department as a whole. Now Gribov's progress was very fast. A year later he received the higher position of the junior scientist. In March 1956 he presented his dissertation for the degree of Candidate of Sciences (analog of $\mathrm{PhD}$ ). It was concerned with interactions of neutrons with nuclei ${ }^{4,5}$. The problem was suggested by K.A. Ter-Martirosyan, but at the presentation of the work he emphasized that methods for calculations were invented by Gribov himself.

After the defence of the dissertation, Shmushkevich and TerMartirosyan organized contacts of Gribov with L.D. Landau and I.Ya. Pomeranchuk. Gribov began to go regularly to Moscow for participating in Landau's seminar. Initially, Landau was sceptical in respect to Gribov (he said: "I know one Gribov, the theatrical actor, and this is enough"). But he rapidly changed his mind. In 1958, when the Scientific 
Council of PTI discussed the next higher position for Gribov, the senior scientist, it received the very favorable recommendation from Landau. Later, Gribov always considered Landau as his main Teacher in theoretical physics.

In 1957, Shmushkevich invited Gribov to give lectures for students of the Leningrad Polytechnical Institute, where he was the Chair of the Theoretical Physics Department. Later, Gribov began to lecture in his alma mater, in the Leningrad University, and in 1968 he became the Professor of the University (the highest scientific degree, Doctor of Sciences, necessary for this, Gribov received in 1964). In parallel, Gribov presented lectures at various Physics Schools, both in the Soviet Union and (later) abroad.

Gribov's scientific work after receiving the Candidate degree became also very active and self-reliant. More and more often he appeared a source of ideas for his colleagues. For instance, in 1958 he investigated three-pion decays of the K-meson. Pair-energy distributions in the decay were shown to depend on pion-pion scattering length ${ }^{6,7}$. For several years after that, the series of papers were published by members of Shmushkevich's group (including Gribov himself) on various inelastic near-threshold reactions. They could allow to obtain information on hadron interactions (e.g., pionpion ones) unreachable in conventional ways. Regretfully, corresponding experimental attempts, as appeared, could not give definite results at those times because of insufficient quality of experimental technique.

One of his further directions of interest (probably, under influence of Pomeranchuk) was high-energy behavior of strong interactions. It was generally assumed for long time to be similar to classical diffraction of light on black screen. Gribov showed that such behavior would be inconsistent with analytic properties of strong-interaction amplitudes ${ }^{8}$. This work of 1961 initiated international interest to his activity. Trying to overcome this diffraction difficulty, Gribov, partly in collaboration with Pomeranchuk, developed reggeology, method of Regge poles and, later, cuts as well. This direction was also actively supported by his colleagues in PTI. He became one of leaders of reggeology not only in the Soviet Union, but in the whole world as well.

At the same time, Gribov tried to use the known quantum field theories as test-grounds for studying their high-energy properties. In Quantum Electrodynamics, as he showed, a special role play the so-called "doublelogarithmic terms". That is why the group of enthusiasts of this approach (G.V. Frolov, V.G. Gorshkov, and L.N. Lipatov first of all) was informally called "the double-logarithmic academy". Their attempts were crowned by papers of Gribov and Lipatov ${ }^{9,10}$ on summation of those "double- 
logarithmic terms", very famous now (they became a basis for studying the evolution of partons).

Gribov could efficiently discuss even such problems in which he was not previously active. For instance, he did not work himself with weak interactions and, in particular, with neutrinos, but when encountered with Pontecorvo's idea of neutrino oscillations Gribov immediately began to construct the corresponding formalism. This resulted in the joint paper of V.N. Gribov and B.M. Pontecorvo ${ }^{11}$, considered now to be classic in the neutrino physics.

There was one more, less known example. In his talk at one of Gribov's seminars, Ya.B. Zeldovich explained that a charged rotating black hole should lose energy by radiation, so its rotation should slow down. When it stops, radiation, according Zeldovich, would stop as well. Here Gribov interrupted him by statement that this is not correct, radiation would continue. Zeldovich waved away this statement, and the seminar talk was continued without discussion of Gribov's suggestion (it seems, however, that their discussions on this problem went on later, but Zeldovich stayed rigid). At that seminar, I restored for myself the lines of Gribov's thought as follows. Gribov, as a physicist, had grown up mainly on quantum physics (in difference with Zeldovich), and he was aware quite well about the Schwinger effect: strong enough electric field, even homogeneous and static (i.e., large gradient of electromagnetic potential), generates electron-positron pairs due to quantum tunneling. Near a black-hole horizon, there is very large gradient of the gravitational potential, which should analogously produce particleantiparticle pairs.

Some time later, Zeldovich again talked at Gribov's seminar, now about the famous paper of Hawking. In particular, Zeldovich said: "Volodya Gribov had tried to assure me that radiation of a black hole should continue, but I did not believe". That is how he lost the interesting and important result.

Gribov ever tried to organize his whole knowledge into some consistent picture. As a rule, such approach is very useful. But not always. It may be not quite adequate if the picture needs strong changes. For Gribov, such was the case of quarks. The idea of quarks was publicized by M. Gell-Mann and G. Zweig in 1964 on the base of resonance spectroscopy. It was further supported by the discovery of scaling in deep-inelastic scattering (in 1968) and, especially, by the unexpected discovery of $J / \psi$ (in 1974). However, Gribov did not believe yet in quarks and, correspondingly, was sceptical in respect to Quantum Chromodynamics (QCD), which arose in 1971-1972. 
It was not a question of taste; for his position Gribov had definite rational arguments. At that time, I asked him once on reasons of his disbelieving in quarks. He answered: "The quarks should be strong-interacting objects; therefore, each of them should be surrounded by a pion cloud. Where is it?" (I should confess, that a clear consistent answer to this question is still absent. Note also that such negative relation to quarks did not prevent Gribov from suggesting to E.M. Levin and L.L. Frankfurt in early days of quarks to compare meson and baryon cross sections in terms of quarks ${ }^{12}$.)

However, in 1976, after discovery of charmed particles, predicted by the quark picture, Gribov changed his mind. He began to study quarks and QCD very intensively. And in 1977 he was able to find a new feature of QCD, known now as the Gribov horizon or Gribov copies ${ }^{13,14}$. It is interesting to note that many-many people worked with QCD to that moment, but existence of the horizon stayed unnoticed. Initially, Gribov hoped that it is just the horizon which determines the origin of confinement of quarks and gluons. But soon he came to conclusion of its insufficiency. Since then he worked hard trying various ways to understand and describe a still unknown mechanism of confinement.

The administrative career of Gribov may look successful. To 1962 the Gatchina site of PTI had the working nuclear reactor and the proton accelerator under construction. There appeared necessity to have there a separate Theoretical Department. Gribov was suggested to organize it. Later, in 1969, after the death of I.M. Shmushktvich, Gribov was returned to the central part of PTI and became the head of its Theoretical Department. In 1971 the Gatchina site of PTI was transformed to be a new institute, Leningrad (now Petersburg) Nuclear Physics Institute (LNPI, now PNPI). All the activity on atomic nuclei and elementary particles was transferred from PTI to the new Institute. Its Theoretical Department was headed, of course, by Gribov. He was a rather good administrator, but, in my opinion, he disliked administrative duties which interfered with his scientific work. Meanwhile, those duties increased along with the Department becoming more populous. In 1980 Gribov moved from LNPI to L.D. Landau Institute of Theoretical Physics in Chernogolovka (near Moscow). There were several reasons for this step, and one of them, as I think, was his wish to diminish necessary administrative duties.

As a physicist, V.N. Gribov was recognized world-wide. He was a speaker at various conferences and schools in the Soviet Union and, sometimes, even abroad. Many foreign physicists, being in the Soviet Union, were eager to visit LNPI for discussions with Gribov. For his scientific 
work Gribov won various prizes, but he was most strongly proud to be the first recipient of the L.D. Landau Prize established in 1971 by the Academy of Sciences of the USSR, which honored his Teacher. In the same year, 1971, he became a member of the American Academy of Arts and Sciences. In the next year, 1972, he was elected (after several unsuccessful attempts) to be a corresponding member of the Academy of Sciences of the USSR. However, official position in respect to him was demonstrated by the fact that Gribov has never been elected to be a full member of the Soviet Academy.

After 1980, Gribov obtained the possibility to be part-time in Budapest, where his second wife, Julia Nyiri, worked in Physics Institute. This made somewhat easier his contacts with West physicists. And after 1990, longer abroad trips became possible, to be a visiting Professor of various Institutes and Universities, both in Europe and in the US.

Active and intensive work of Gribov was unexpectedly interrupted by the acute stroke in 1997 during one of scientific conferences. He was taken to a hospital and, after stabilization of his state, was transported to another hospital, in Budapest. Even there he tried to continue investigations of confinement. The medical treatment looked successful, and the physicians planned that Gribov would be able soon to go home. However, on August 13, 1997 he passed away. His grave in Budapest is marked by the memorial that reminds a beautiful fading flower, with the simple epitaph

\section{VLADIMIR GRIBOV \\ FIZIKUS \\ 1930-1997}

on the basement.

After Gribov's death, his works have not been forgotten. Just opposite, many of his papers are republished. His lectures. which were mainly written up in Russian, but not always published, are now collected, translated into English and published as books. Therefore, his results become available and well-known even for younger generations of physicists. If judging by references, the most famous and operative of those results seem to be the Gribov copies (they are especially essential now in lattice calculations) and DGLAP equations for evolution of partons (here G stays just for Gribov). There established are various stipends and prizes called by Gribov's name.

And yet there are Gribov's last papers or even notes. They are mainly not completed or, at least, not quite understood by the world community. Meanwhile, they tried various ways to solve the problem of confinement, one of the hottest problems in strong interactions. In some sense the situation 
may be similar to the fate of Einstein's last ideas. During his life, they looked to be out of mainstream, but now many of them feed new theoretical approaches. Such future is not excluded also for Gribov's last ideas. Look forward...

\section{Acknowledgments}

This work is supported by the Russian Science Foundation (Grant No.1422-00281).

\section{References}

1. V. N. Gribov, "On the interaction of two electrons", Vest. Leningrad. Gos. Univ. 3, 10 (1953) [in Russian].

2. L. E. Gurevich, V. N. Gribov, "Dielectric losses in ionic dielectrics in strong electric fields", Zh. Eksp. Teor. Fiz. 29, 629 (1955) [Sov. Phys. JETP 2, 565 (1956)].

3. V. N. Gribov, L. E. Gurevich, "On the theory of the stability of a layer located at the superadiabatic temperature gradient in the gravitational field", $Z h$. Eksp. Teor. Fiz. 31, 854 (1956) [Sov. Phys. JETP 4, 720 (1957)].

4. V. N. Gribov, "Effect of diffuseness of the nuclear boundary on neutron scattering", Zh. Eksp. Teor. Fiz. 32, 647 (1957) [Sov. Phys. JETP 5, 537 (1957)].

5. V. N. Gribov, "Excitation of rotational states in the interaction berween neutrons and nuclei", Zh. Eksp. Teor. Fiz. 32, 842 (1957) [Sov. Phys. JETP 5, 688 (1957)].

6. V. N. Gribov, "Angular distribution in reactions involving the formation of three low energy particles, with application to $\tau^{+}$meson decay", Nucl. Phys., 5, 653 (1958).

7. V. N. Gribov, "Angular distribution in the reactions $K^{+} \rightarrow 2 \pi^{+}+\pi^{-}$and $K^{+} \rightarrow 2 \pi^{0}+\pi^{+}$", Zh. Eksp. Teor. Fiz. 34, 749 (1958) [Sov. Phys. JETP 7, $514(1958)]$.

8. V. N. Gribov, "Asymptotic behaviour of the scattering amplitude at high energies", Nucl. Phys., 22, 249 (1961).

9. V. N. Gribov and L. N. Lipatov, "Deep inelastic ep scattering in perturbation theory", Yad.Fiz. 15, 781 (1972) [Sov. J. Nucl. Phys. 15, 438 (1972)].

10. V. N. Gribov and L. N. Lipatov, " $e^{+} e^{-}$pair annihilation and deep inelastiv ep scattering in perturbation theory", Yad.Fiz. 15, 1218 (1972) [Sov. J. Nucl. Phys. 15, 675 (1972)].

11. V. N. Gribov and B. Pontecorvo, "Neutrino astronomy and lepton charge", Phys.Lett. 493 (1969).

12. E. M. Levin and L. L. Frankfurt, "The quark hypothesis and relations between cross sections at high energies", Pisma ZhETF 2, 105 (1965) [JETP Lett. 2, 65 (1965)].

13. V. N. Gribov, "Instability of nonabelian gauge theories and impossibility of choice of Coulomb gauge", Proceedings of the 12th LNPI Winter School on 
Nuclear and Elementary Particle Physics, v.1, pp. 147-162, Leningrad 1977 (in Russian); for English translation, see SLAC-TRANS-0176.

14. V. N. Gribov, "Quantization of nonabelian gauge theories", Nucl.Phys. B139, 1 (1978). 